\title{
Deviations for Jumping Times of a Branching Process Indexed by a Poisson Process
}

\author{
Yanhua Zhang $\mathbb{D i D}^{1}$ and Zhenlong Gao $\mathbb{D}^{2}$ \\ ${ }^{1}$ School of Mathematical Sciences, Qufu Normal University, Qufu 273165, China \\ ${ }^{2}$ School of Statistics, Qufu Normal University, Qufu 273165, China \\ Correspondence should be addressed to Zhenlong Gao; gzlkygz@163.com
}

Received 25 January 2019; Accepted 9 May 2019; Published 23 May 2019

Academic Editor: Fazal M. Mahomed

Copyright (c) 2019 Yanhua Zhang and Zhenlong Gao. This is an open access article distributed under the Creative Commons Attribution License, which permits unrestricted use, distribution, and reproduction in any medium, provided the original work is properly cited.

\begin{abstract}
Consider a continuous time process $\left\{Y_{t}=Z_{N_{t}}, t \geq 0\right\}$, where $\left\{Z_{n}\right\}$ is a supercritical Galton-Watson process and $\left\{N_{t}\right\}$ is a Poisson process which is independent of $\left\{Z_{n}\right\}$. Let $\tau_{n}$ be the $n$-th jumping time of $\left\{Y_{t}\right\}$, we obtain that the typical rate of growth for $\left\{\tau_{n}\right\}$ is $n / \lambda$, where $\lambda$ is the intensity of $\left\{N_{t}\right\}$. Probabilities of deviations $\left\{\left|n^{-1} \tau_{n}-\lambda^{-1}\right|>\delta\right\}$ are estimated for three types of positive $\delta$.
\end{abstract}

\section{Statements of the Main Results}

The model of Poisson randomly indexed branching process (PRIBP) $\left\{Y_{t}=Z_{N_{t}}, t \geq 0\right\}$ was introduced by [1] to study the evolution of stock prices and its statistical investigation was done in [2].

In a recent manuscript [3] the authors there consider the asymptotic properties of $\log Y_{t}$. Let $\left\{p_{k}, k \geq 0\right\}$ be the offspring distribution of the branching process with mean $m=\sum_{k} k p_{k} \in(1, \infty)$; we distinguish between the Shröder case and the Böttcher case depending on whether $p_{0}+p_{1}>0$ or $p_{0}+p_{1}=0$. In Böttcher case, it was proved in [3] that $\log Y_{t}$ have similar asymptotic results to the Poisson process $\left\{N_{t}\right\}$. But differences appeared in Shröder case; see [4]. For subcritical and critical PRIBP, one can see $[5,6]$ for details.

In this paper, we deal with the asymptotic theory for the jumping times of PRIBP defined as follows. For any $\omega$, define

$$
\tau_{\infty}(\omega)=\inf \left\{t: t>0 ; \lim _{s \uparrow t} Y_{s}(\omega)=\infty\right\}
$$

where $\inf \emptyset=\infty$; then

$$
\left\{\tau_{\infty}<\infty\right\}=\bigcup_{l=1}^{\infty}\left\{\tau_{\infty}<l\right\} .
$$

Note that $\left\{Z_{n}\right\}$ is independent of $\left\{N_{t}\right\}$; one has

$$
\begin{aligned}
P\left(\tau_{\infty}<l\right) & \leq P\left(Y_{l}=\infty\right)=\sum_{n} P\left(Z_{n}=\infty\right) P\left(N_{l}=n\right) \\
& =0
\end{aligned}
$$

and thus $P\left(\tau_{\infty}=\infty\right)=1$. Define $\tau_{0}=0$ and $\left\{\tau_{n}, n \geq 1\right\}$ as the successive times of jump of the PRIBP $\left\{Y_{t}, t \geq 0\right\}$.

In Böttcher case, the jumping times of $\left\{Y_{t}\right\}$ coincide with that of $\left\{N_{t}\right\}$. Let $\left\{T_{n}\right\}$ be the successive times of jump of $\left\{N_{t}\right\}$; then both $\tau_{n}$ and $T_{n}$ have a gamma distribution with parameters $n$ and $\lambda$. But when $p_{0}+p_{1}>0$, at the jumping time of $N_{t}$, PRIBP can have no jump, since an individual can replicate himself at this time. So the jumping times of $\left\{Y_{t}\right\}$ are likely to be delayed; see Figure 1 for example. In the path of Figure $1, \tau_{1}=T_{1}$ and $\tau_{2}=T_{3}, \cdots$.

Although $\tau_{n} \geq T_{n}$ for all $n$, the growth rate of $\tau_{n}$ is not too fast as that of $T_{n}$. In fact, the typical growth rate of $T_{n}$ is $n / \lambda$ by the law of large numbers and we can show that the typical growth rate of $\tau_{n}$ is

$$
\sum_{k=1}^{n} \frac{1}{\lambda\left(1-p_{1}^{k}\right)} \leq \frac{n}{\lambda}+\frac{1}{\lambda\left(1-p_{1}\right)^{2}},
$$

and see the proof of Theorem 1. Thus, for almost all the path of Shröder case PRIBP, $\tau_{n} / n$ has a limit $\lambda^{-1}$ when $n \longrightarrow \infty$. 


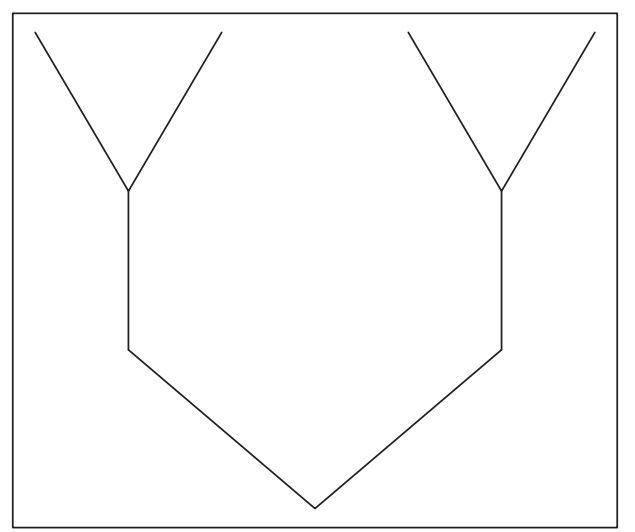

FIGURE 1: A path of Shröder case branching process.

In the rest of this paper, we always assume that our branching process belongs to the Shröder case, $p_{0}=0$ and $Z_{0}=1$.

We are interested in the decay rates about the probabilities of

$$
\left\{\omega:\left|\frac{\tau_{n}(\omega)}{n}-\frac{1}{\lambda}\right|>\delta\right\}
$$

for some positive $\delta$. Typically, there are three classes of $\delta$ to be chosen.

The first one is that $\delta=a \sqrt{n}$ for some fixed $a>0$. In this case, the event in (5) is said to be a normal deviation event. The decay rate of its probability can be characterized by the following central limit theorem.

Theorem 1. $\left\{\tau_{n}\right\}$ satisfies the law of large number and the central limit theorem; that is, $\tau_{n} / n \stackrel{\text { a.e. }}{\longrightarrow} \lambda^{-1}$ and $\lambda \sqrt{n}\left(\tau_{n} / n-\right.$ $\left.\lambda^{-1}\right) \stackrel{d}{\rightarrow} N(0,1)$ when $n \longrightarrow \infty$, where $N(0,1)$ is standard normal distribution.

Next, if $\delta=a$ for some fixed $a>0$, the event in (5) is said to be a large deviation event whose probability has an exponential convergence rate by the following large deviation principle.

Theorem 2 (LDP). For any measurable subset $B$ of $\mathbb{R}$,

$$
\begin{aligned}
-\inf _{x \in B^{o}} \Lambda^{*}(x) & \leq \liminf _{n \rightarrow \infty} \frac{1}{n} \log P\left(\frac{\tau_{n}}{n} \in B\right) \\
& \leq \limsup _{n \rightarrow \infty} \frac{1}{n} \log P\left(\frac{\tau_{n}}{n} \in B\right) \\
& \leq-\inf _{x \in \bar{B}} \Lambda^{*}(x),
\end{aligned}
$$

where $B^{o}$ denotes the interior of $B, \bar{B}$ its closure, and

$$
\Lambda^{*}(x)= \begin{cases}\lambda\left(1-p_{1}\right) x+\log p_{1}, & x \geq\left(\lambda p_{1}\right)^{-1} ; \\ \lambda x-\log (\lambda x)-1, & \left(\lambda p_{1}\right)^{-1}>x>0 ; \\ +\infty, & x \leq 0 .\end{cases}
$$

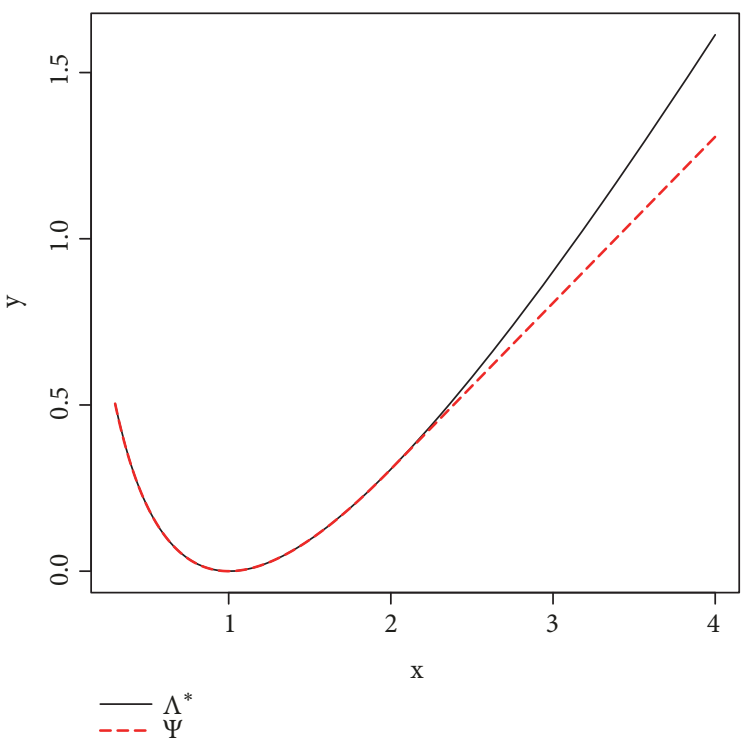

Figure $2: \lambda=1, p_{1}=1 / 2$.

Remark. By Cramér's theorem (see Theorem 2.2.3 of [7]), $T_{n} / n$ satisfies the large deviation principle with rate function

$$
\Psi(x)= \begin{cases}\lambda x-\log (\lambda x)-1, & x>0 ; \\ +\infty, & x \leq 0 .\end{cases}
$$

By Theorem 2 , the rate function of $\tau_{n} / n$ coincides with that of $T_{n} / n$ for $x \leq\left(\lambda p_{1}\right)^{-1}$, but differences appeared for large $x$; see Figure 2 for example.

If $\delta=\delta_{n} \longrightarrow \infty$ and $\delta_{n}=o(\sqrt{n})$ as $n \longrightarrow \infty$, we call the event in (5) a moderate deviation event. Let $\left\{a_{n}, n \geq 0\right\}$ be a family of positive numbers satisfying

$$
\begin{gathered}
\frac{a_{n}}{n} \longrightarrow 0 \\
\text { and } \frac{a_{n}}{\sqrt{n}} \longrightarrow \infty
\end{gathered}
$$

as $n \longrightarrow \infty$.

As in the case of large deviation principle, based on the Gärtner-Ellis theorem (see [7], page 44), we have the following moderate deviation principle.

Theorem 3 (MDP). For any measurable subset $B$ of $R$,

$$
\begin{aligned}
-\inf _{x \in B^{\circ}} \frac{\lambda^{2} x^{2}}{2} & \leq \liminf _{n \rightarrow \infty} \frac{n}{a_{n}^{2}} \log P\left(\frac{\tau_{n}-\lambda^{-1} n}{a_{n}} \in B\right) \\
& \leq \limsup _{t \rightarrow \infty} \frac{n}{a_{n}^{2}} \log P\left(\frac{\tau_{n}-\lambda^{-1} n}{a_{n}} \in B\right) \\
& \leq-\inf _{x \in \bar{B}} \frac{\lambda^{2} x^{2}}{2} .
\end{aligned}
$$

The rest of the paper is organized as follows. In Section 2, we prove the law of the large number and the central limit 
theorem. Section 3 is devoted to the proof of the large deviation principle. In Section 4, the moderate deviation principle is obtained. Basic facts on Gärtner-Ellis theorem are given in the Appendix.

\section{The Law of Large Number and the Cental Limit Theorem}

Proposition 4. PRIBP is a homogenous continuous time Markov chain in which $Q$ matrix satisfies $q_{i}=\lambda\left(1-p_{1}^{i}\right)$ for all $i \geq 1$.

Proof. For any nonnegative real numbers $t_{0}<t_{1}<\cdots<$ $t_{n-1}<s<t+s$ and nonnegative integers $i_{0} \leq i_{1} \leq \cdots \leq i_{n-1} \leq$ $i \leq j$, define

$$
A=\left\{Y(s)=i, Y\left(t_{n-1}\right)=i_{n-1}, \ldots, Y\left(t_{0}\right)=i_{0}\right\}
$$

For any nonnegative integers $k_{0} \leq k_{1} \leq \cdots \leq k_{n-1} \leq k_{s} \leq$ $k_{t+s}$, define

$$
\begin{aligned}
& B\left(k_{0}, \ldots, k_{n-1}, k_{s}, k_{t+s}\right)=\left\{N\left(t_{0}\right)=k_{0}, \ldots, N\left(t_{n-1}\right)\right. \\
& \left.\quad=k_{n-1}, N(s)=k_{s}, N(t+s)=k_{t+s}\right\} .
\end{aligned}
$$

Since Poisson process $\left\{N_{t}\right\}$ is independent of the GaltonWatson process $\left\{Z_{n}\right\}$,

$$
\begin{aligned}
& P(Y(t+s)=j \mid A) \\
& =\sum_{k_{0}, \ldots, k_{n-1}, k_{s}, k_{t+s}} P\left(B\left(k_{0}, \ldots, k_{n-1}, k_{s}, k_{t+s}\right)\right) \\
& \quad \cdot P\left(Z_{k_{t+s}}=j \mid Z_{k_{s}}=i, Z_{k_{n-1}}=i_{n-1}, \ldots, Z_{k_{0}}=i_{0}\right) .
\end{aligned}
$$

Note that the Galton-Watson process is a Markov chain with $n$ - step transition probabilities $P_{n}(i, j)$, and summing $k_{0}, \ldots, k_{n-1}$, one has

$$
\begin{aligned}
P( & Y(t+s)=j \mid A) \\
& =\sum_{k_{s}, k_{t+s}} P\left(N(s)=k_{s}, N(t+s)=k_{t+s}\right) \\
& \cdot P\left(Z_{k_{t+s}}=j \mid Z_{k_{s}}=i\right) \\
& =\sum_{k_{s}, k_{t+s}} P\left(N(s)=k_{s}, N(t+s)=k_{t+s}\right) P_{k_{t+s}-k_{s}}(i, j) \\
& =E\left(P_{N(t+s)-N(s)}(i, j)\right)=E\left(P_{N(t)}(i, j)\right) .
\end{aligned}
$$

Similarly,

$$
\begin{aligned}
& P(Y(t+s)=j \mid Y(s)=i)=E\left(P_{N(t)}(i, j)\right) \\
& \quad=P(Y(t+s)=j \mid A),
\end{aligned}
$$

which means that PRIBP is a homogenous continuous time Markov chain.
Next, note that $N_{t}$ has a Poisson distribution with parameter $\lambda t>0$; we have

$$
\begin{aligned}
E\left(P_{N(t)}(i, i)\right)= & e^{-\lambda t}+p_{1}^{i} \lambda t e^{-\lambda t} \\
& +\sum_{n \geq 2} p_{1}^{n i} P(N(t)=n),
\end{aligned}
$$

which implies $q_{i}=\lambda\left(1-p^{i}\right)$.

Proof of Theorem 1. Define $X_{n}(\omega)=Y\left(\tau_{n}(\omega)\right)(\omega)$; then $\left\{X_{n}\right\}$ is a homogeneous discrete-time Markov chain. Define $\rho_{n}=$ $\tau_{n}-\tau_{n-1}$ for $n \geq 1$; then the conditional distribution of $\rho_{n}$ relative to $X_{1}, X_{2}, \ldots, X_{n-1}$ equals exponential distribution with parameter $q_{X_{n-1}}$, where $q_{i}=\lambda\left(1-p^{i}\right)$, see page 259 of [8] for example. So

$$
\begin{aligned}
\tau_{n}^{\prime} & :=\tau_{n}-\sum_{k=1}^{n} \frac{1}{\lambda\left(1-p_{1}^{X_{k-1}}\right)} \\
& =\sum_{k=1}^{n}\left(\rho_{k}-\frac{1}{\lambda\left(1-p_{1}^{X_{k-1}}\right)}\right)
\end{aligned}
$$

is a square-integrable martingale adapted to the $\sigma$ - fields $\sigma\left\{X_{1}, \ldots, X_{n}\right\}$. Consequently, there exists a random variable $Z$ such that $\tau_{n}^{\prime} \stackrel{\text { a.e. }}{\longrightarrow} Z$, see page 2 of [9]. Note that $X_{n-1} \geq n$; one has

$$
\begin{aligned}
\frac{n}{\lambda} & \leq \sum_{k=1}^{n} \frac{1}{\lambda\left(1-p_{1}^{X_{k-1}}\right)} \leq \sum_{k=1}^{n} \frac{1}{\lambda\left(1-p_{1}^{k}\right)} \\
& \leq \frac{n}{\lambda}+\frac{1}{\lambda\left(1-p_{1}\right)^{2}}
\end{aligned}
$$

which implies that $\tau_{n} / n \stackrel{\text { a.e. }}{\longrightarrow} \lambda^{-1}$ as $n \longrightarrow \infty$.

Next, we prove $\lambda \tau_{n}^{\prime} / \sqrt{n} \stackrel{d}{\rightarrow} N(0,1)$. Let $\eta_{n, i}=$ $\lambda n^{-1 / 2}\left[\rho_{i}-\left(\lambda\left(1-p_{1}^{X_{i-1}}\right)\right)^{-1}\right], \mathscr{F}_{i-1}$ be the $\sigma$ - field generated by $X_{1}, \ldots, X_{i-1}$; by Hölder's inequality, one has

$$
\begin{aligned}
& E\left(\eta_{n, i}^{2} I_{\left\{\left|\eta_{n, i}\right|>\epsilon\right\}} \mid \mathscr{F}_{i-1}\right) \\
& \quad \leq\left[E\left(\left|\eta_{n, i}\right|^{3} \mid \mathscr{F}_{i-1}\right)\right]^{2 / 3}\left[E\left(I_{\left\{\left|\eta_{n, i}\right|>\epsilon\right\}} \mid \mathscr{F}_{i-1}\right)\right]^{1 / 3},
\end{aligned}
$$

where $I_{A}$ is the indicator function of $A$.

Note that the conditional distribution of $\rho_{n}$ relative to $\rho_{1}, \ldots, \rho_{n-1}$ equals exponential distribution with parameter $\lambda\left(1-p_{1}^{X_{n-1}}\right)$; one has

$$
\begin{aligned}
& E\left(\left|\eta_{n, i}\right|^{3} \mid \mathscr{F}_{i-1}\right) \\
& \quad=(\lambda n)^{-3 / 2} E\left(\left|\rho_{i}-\left(\lambda\left(1-p_{1}^{X_{i-1}}\right)\right)^{-1}\right|^{3} \mid \mathscr{F}_{i-1}\right) \\
& \quad \leq 4 n^{-3 / 2}\left(\lambda^{1 / 2}\left(1-p_{1}^{X_{i-1}}\right)\right)^{-3} \\
& \quad \leq 4 n^{-3 / 2}\left(\lambda^{1 / 2}\left(1-p_{1}\right)\right)^{-3} \text { a.s.. }
\end{aligned}
$$


According to Markov's inequality,

$$
\begin{aligned}
E\left(I_{\left\{\left|\eta_{n, i}\right|>\epsilon\right\}} \mid \mathscr{F}_{i-1}\right) & \leq \epsilon^{-2} E\left(\eta_{n, i}^{2} \mid \mathscr{F}_{i-1}\right) \\
& =\left(n \epsilon^{2}\left(1-p_{1}^{X_{i-1}}\right)^{2}\right)^{-1} \\
& \leq\left(n \epsilon^{2}\left(1-p_{1}\right)^{2}\right)^{-1} \text { a.s.. }
\end{aligned}
$$

By formulas (19)-(21) we have

$$
\sum_{i=1}^{n} E\left(\eta_{n, i}^{2} I_{\left\{\left|\eta_{n, i}\right|>\epsilon\right\}} \mid \mathscr{F}_{i-1}\right) \leq C n^{-1 / 3} \text { a.e. }
$$

for some positive constant $C$. Similarly,

$$
\sum_{i=1}^{n} E\left(\eta_{n, i}^{2} \mid \mathscr{F}_{i-1}\right)=\frac{1}{n} \sum_{i=1}^{n}\left(1-p_{1}^{X_{i-1}}\right)^{-2} \stackrel{\text { a.e. }}{\longrightarrow} 1 .
$$

According to (22), (23), and Corollary 3.1 of [9], one has $\lambda \tau_{n}^{\prime} / \sqrt{n} \stackrel{d}{\rightarrow} N(0,1)$. Note that

$$
\begin{aligned}
& \left|\frac{\lambda \tau_{n}^{\prime}}{\sqrt{n}}-\lambda \sqrt{n}\left(\frac{\tau_{n}}{n}-\lambda^{-1}\right)\right| \\
& \quad=\left|\sqrt{n}-\frac{1}{\sqrt{n}} \sum_{i=1}^{n}\left(1-p_{1}^{X_{i-1}}\right)^{-1}\right| \leq\left[\left(1-p_{1}\right)^{2} \sqrt{n}\right]^{-1} \longrightarrow
\end{aligned}
$$

0

and the central limit theorem follows from Theorem 6 in page 39 of [10].

\section{Large Deviation Principle}

Let us begin with some lemmas to show the conditions of Gärtner-Ellis Theorem (see Appendix) are satisfied. Define $\Lambda_{n}(\theta)=\log E\left[e^{\theta \tau_{n} / n}\right]$.

Lemma 5. For any $\theta \in R, \Lambda(\theta):=\lim _{n \rightarrow \infty}(1 / n) \Lambda_{n}(n \theta)$ exists and satisfies

$$
\Lambda(\theta)= \begin{cases}\log \lambda-\log (\lambda-\theta), & \theta<\lambda\left(1-p_{1}\right) ; \\ +\infty, & \theta \geq \lambda\left(1-p_{1}\right) .\end{cases}
$$

Particularly, $0 \in D_{\Lambda}:=\{\theta: \Lambda(\theta)<\infty\}$.

Proof. Note that $\tau_{n}=\rho_{1}+\cdots+\rho_{n}$, where the conditional distribution of $\rho_{n}$ relative to $\rho_{1}, \ldots, \rho_{n-1}$ is the same as that relative to $X_{0}, X_{1}, \ldots, X_{n-1}$ and equals exponential distribution with parameter $\lambda\left(1-p_{1}^{X_{n-1}}\right)$ (see page 259 of [8]); one has

$$
\begin{aligned}
E\left[e^{\theta \tau_{n}}\right] & =E\left[e^{\theta \sum_{i=1}^{n} \rho_{i}}\right] \\
& =E\left[E\left[e^{\theta \sum_{i=1}^{n} \rho_{i}} \mid \rho_{1}, \ldots, \rho_{n-1}\right]\right] \\
& =E\left[e^{\theta \sum_{i=1}^{n-1} \rho_{i}} E\left(e^{\theta \rho_{n}} \mid \rho_{1}, \ldots, \rho_{n-1}\right)\right] \\
& =E\left[e^{\theta \sum_{i=1}^{n-1} \rho_{i}} E\left(e^{\theta \rho_{n}} \mid X_{0}, \ldots, X_{n-1}\right)\right] .
\end{aligned}
$$

If $\theta<\lambda\left(1-p_{1}\right)$, we have

$$
E\left(e^{\theta \rho_{n}} \mid X_{0}, \ldots, X_{n-1}\right)=\frac{\lambda\left(1-p_{1}^{X_{n-1}}\right)}{\lambda\left(1-p_{1}^{X_{n-1}}\right)-\theta} .
$$

If $\theta \leq 0$, note that $X_{n-1} \geq n$; by (27) one has

$$
\frac{\lambda\left(1-p_{1}^{n}\right)}{\lambda\left(1-p_{1}^{n}\right)-\theta} \leq E\left(e^{\theta \rho_{n}} \mid X_{0}, \ldots, X_{n-1}\right) \leq \frac{\lambda}{\lambda-\theta} .
$$

By (26), (28) and induction, we have

$$
\prod_{i=1}^{n} \frac{\lambda\left(1-p_{1}^{i}\right)}{\lambda\left(1-p_{1}^{i}\right)-\theta} \leq E\left[e^{\theta \tau_{n}}\right] \leq \frac{\lambda^{n}}{(\lambda-\theta)^{n}}
$$

which means $\Lambda(\theta)=\log \lambda-\log (\lambda-\theta)$.

If $0<\theta<\lambda\left(1-p_{1}\right)$, note that $X_{n-1} \geq n$; by (27) one has

$$
\frac{\lambda}{\lambda-\theta} \leq E\left(e^{\theta \rho_{n}} \mid X_{0}, \ldots, X_{n-1}\right) \leq \frac{\lambda\left(1-p_{1}^{n}\right)}{\lambda\left(1-p_{1}^{n}\right)-\theta} .
$$

We can get $\Lambda(\theta)=\log \lambda-\log (\lambda-\theta)$ similarly.

If $\theta \geq \lambda\left(1-p_{1}\right)$, note that $X_{0}=Z_{0}=1$ and the conditional distribution of $\rho_{1}$ relative to $X_{0}$ equals exponential distribution with parameter $\lambda\left(1-p_{1}\right)$; one has

$$
E\left[e^{\theta \tau_{n}}\right] \geq E\left[e^{\theta \rho_{1}}\right]=E\left[E\left(e^{\theta \rho_{1}} \mid X_{0}\right)\right]=+\infty,
$$

for all $n \geq 1$. Thus $\Lambda(\theta)=+\infty$.

Lemma 6. Let $\Lambda^{*}$ be the Fenchel-Legendre transform of $\Lambda$; then

$$
\begin{aligned}
\Lambda^{*}(x) & =\sup _{\theta \in R}\{\theta x-\Lambda(\theta)\} \\
& = \begin{cases}\lambda\left(1-p_{1}\right) x+\log p_{1}, & x \geq\left(\lambda p_{1}\right)^{-1} \\
\lambda x-\log (\lambda x)-1, & \left(\lambda p_{1}\right)^{-1}>x>0 ; \\
+\infty, & x \leq 0 .\end{cases}
\end{aligned}
$$

In addition, the set of exposed points (see Appendix) of $\Lambda^{*}$ is $\mathscr{E} \supset(0,+\infty)$.

Proof. By Lemma 5, if $x \leq 0$,

$$
\begin{aligned}
\Lambda^{*}(x) & =\sup _{\theta<\lambda\left(1-p_{1}\right)}\{\theta x-\log \lambda+\log (\lambda-\theta)\} \\
& =\lim _{\theta \rightarrow-\infty}(\theta x-\log \lambda+\log (\lambda-\theta))=+\infty .
\end{aligned}
$$

Next, if $\left(\lambda p_{1}\right)^{-1}>x>0$, then $\lambda-x^{-1}<\lambda\left(1-p_{1}\right)$ and

$$
\begin{aligned}
\Lambda^{*}(x) & =\sup _{\theta<\lambda\left(1-p_{1}\right)}\{\theta x-\log \lambda+\log (\lambda-\theta)\} \\
& \stackrel{\theta_{0}=\lambda-x^{-1}}{=} \theta_{0} x-\log \lambda+\log \left(\lambda-\theta_{0}\right) \\
& =\lambda x-\log (\lambda x)-1 .
\end{aligned}
$$


Finally, if $x \geq\left(\lambda p_{1}\right)^{-1}$, then $\lambda-x^{-1} \geq \lambda\left(1-p_{1}\right)$. Note that

$$
\frac{d(\theta x-\log \lambda+\log (\lambda-\theta))}{d \theta}=x-\frac{1}{\lambda-\theta}>0
$$

for all $\theta<\lambda\left(1-p_{1}\right)$; we have

$$
\begin{aligned}
\Lambda^{*}(x) & =\sup _{\theta<\lambda\left(1-p_{1}\right)}\{\theta x-\log \lambda+\log (\lambda-\theta)\} \\
\theta_{0}=\lambda\left(1-p_{1}\right) & \stackrel{=}{=} \theta_{0} x-\log \lambda+\log \left(\lambda-\theta_{0}\right) \\
& =\lambda\left(1-p_{1}\right) x+\log p_{1} .
\end{aligned}
$$

Equation (32) follows from (33), (34), and (36).

In addition, for any $\theta<\lambda, \Lambda^{\prime}(\theta)=(\lambda-\theta)^{-1}$, so the range of $\Lambda^{\prime}(\theta)$ for $\theta<\lambda$ is $(0,+\infty)$, which is a subset of $\mathscr{E}$, the set of exposed points of $\Lambda^{*}$; see Lemma 2.3.9 of [7].

Proof of Theorem 2. Note that for any $x \leq 0, \Lambda^{*}(x)=+\infty$ and the set of exposed points $\mathscr{E} \supset(0,+\infty)$, then for any open set G,

$$
\inf _{x \in G \cap \mathscr{E}} \Lambda^{*}(x)=\inf _{x \in G \cap(0,+\infty)} \Lambda^{*}(x)=\inf _{x \in G} \Lambda^{*}(x) .
$$

Consequently, Theorem 2 follows from Lemma 5, Lemma 6, and the Gärtner-Ellis theorem (see Appendix).

\section{Moderate Deviation Principle}

In this section, we deal with the proof of Theorem 3. Define

$$
\Lambda_{n}(\theta)=\log E\left[\exp \left\{\theta \cdot \frac{\tau_{n}-\lambda^{-1} n}{a_{n}}\right\}\right] .
$$

Lemma 7. For each $\theta \in R$, one has,

$$
\Delta(\theta)=\lim _{n \longrightarrow \infty} \frac{n}{a_{n}^{2}} \Lambda_{n}\left(\frac{a_{n}^{2}}{n} \theta\right)=\frac{\theta^{2}}{2 \lambda^{2}} .
$$

Particularly, $0 \in D_{\Delta}:=\{\theta: \Delta(\theta)<\infty\}$. In addition, let $\Delta^{*}$ be the Fenchel-Legendre transform of $\Delta$; then

$$
\Delta^{*}(x)=\frac{\lambda^{2} x^{2}}{2}
$$

and the set of exposed points of $\Delta^{*}$ is $\mathscr{F}=R$.

Proof. For any $\theta \in R$, we have

$$
\begin{gathered}
\Lambda_{n}\left(\frac{a_{n}^{2}}{n} \theta\right)=\log E\left[\exp \left\{\frac{a_{n}^{2}}{n} \theta \cdot \frac{\tau_{n}-\lambda^{-1} n}{a_{n}}\right\}\right] \\
=\log E\left[\exp \left\{\frac{a_{n} \theta}{n}\left(\tau_{n}-\sum_{i=1}^{n} \frac{1}{\lambda\left(1-p_{1}^{X_{i-1}}\right)}\right)\right\}\right. \\
\left.\cdot \exp \left\{\frac{a_{n} \theta}{n} \cdot \sum_{i=1}^{n} \frac{p_{1}^{X_{i-1}}}{\lambda\left(1-p_{1}^{X_{i-1}}\right)}\right\}\right] .
\end{gathered}
$$

For any $\theta \geq 0$, note that $X_{i-1} \geq i$; one has

$$
\begin{aligned}
0 & \leq \frac{a_{n} \theta}{n} \cdot \sum_{i=1}^{n} \frac{p_{1}^{X_{i-1}}}{\lambda\left(1-p_{1}^{X_{i-1}}\right)} \leq \frac{a_{n} \theta}{n} \cdot \sum_{i=1}^{n} \frac{p_{1}^{i}}{\lambda\left(1-p_{1}^{i}\right)} \\
& \leq \frac{a_{n} \theta}{n} \frac{1}{\lambda\left(1-p_{1}\right)^{2}} .
\end{aligned}
$$

Similarly, for $\theta<0$, the above inequality should be reversed. Thus, by (41), if $\Delta(\theta)$ exists, one has

$$
\begin{aligned}
\Delta(\theta) & =\lim _{n \longrightarrow \infty} \frac{n}{a_{n}^{2}} \log E \\
\cdot & {\left[\exp \left\{\frac{a_{n} \theta}{n}\left(\tau_{n}-\sum_{i=1}^{n} \frac{1}{\lambda\left(1-p_{1}^{X_{i-1}}\right)}\right)\right\}\right] . }
\end{aligned}
$$

Define $\beta_{i}=\rho_{i}-\left(\lambda\left(1-p_{1}^{X_{i-1}}\right)\right)^{-1}$; then

$$
\tau_{n}-\sum_{i=1}^{n} \frac{1}{\lambda\left(1-p_{1}^{X_{i-1}}\right)}=\sum_{i=1}^{n} \beta_{i}
$$

Note that the conditional distribution of $\rho_{n}$ relative to $\rho_{1}, \ldots, \rho_{n-1}$ is the same as that relative to $X_{0}, X_{1}, \ldots, X_{n-1}$ and equals exponential distribution with parameter $\lambda\left(1-p_{1}^{X_{n-1}}\right)$; one has

$$
\begin{aligned}
& E\left[\exp \left\{\frac{a_{n} \theta}{n} \sum_{i=1}^{n} \beta_{i}\right\}\right] \\
& =E\left[\exp \left\{\frac{a_{n} \theta}{n} \sum_{i=1}^{n-1} \beta_{i}\right\} E\left[\exp \left\{\frac{a_{n} \theta}{n} \beta_{n}\right\} \mid \mathscr{F}_{n-1}\right]\right],
\end{aligned}
$$

where $\mathscr{F}_{i-1}$ is the $\sigma$ - field generated by $X_{1}, \ldots, X_{i-1}$. After a simple calculation,

$$
\begin{aligned}
& E\left[\exp \left\{\frac{a_{n} \theta}{n} \beta_{i}\right\} \mid \mathscr{F}_{i-1}\right] \\
& \quad=\exp \left\{\frac{-\theta a_{n}}{n \lambda\left(1-p_{1}^{X_{i-1}}\right)}\right\} \cdot \frac{\lambda\left(1-p_{1}^{X_{i-1}}\right)}{\lambda\left(1-p_{1}^{X_{i-1}}\right)-a_{n} \theta / n}
\end{aligned}
$$

For $\theta>0$,

$$
\begin{aligned}
& \exp \left\{\frac{-\theta a_{n}}{n \lambda\left(1-p_{1}^{i}\right)}\right\} \cdot \frac{\lambda}{\lambda-a_{n} \theta / n} \\
& \leq E\left[\exp \left\{\frac{a_{n} \theta}{n} \beta_{i}\right\} \mid \mathscr{F}_{i-1}\right] \\
& \quad \leq \exp \left\{\frac{-\theta a_{n}}{n \lambda}\right\} \cdot \frac{\lambda\left(1-p_{1}^{i}\right)}{\lambda\left(1-p_{1}^{i}\right)-a_{n} \theta / n} .
\end{aligned}
$$


According to (45)-(47) and induction, we obtain

$$
\begin{aligned}
I_{n}(\theta) & :=\prod_{i=1}^{n}\left[\exp \left\{\frac{-\theta a_{n}}{n \lambda\left(1-p_{1}^{i}\right)}\right\} \cdot \frac{\lambda}{\lambda-a_{n} \theta / n}\right] \\
& \leq E\left[\exp \left\{\frac{a_{n} \theta}{n} \sum_{i=1}^{n} \beta_{i}\right\}\right] \\
& \leq \prod_{i=1}^{n}\left[\exp \left\{\frac{-\theta a_{n}}{n \lambda}\right\} \cdot \frac{\lambda\left(1-p_{1}^{i}\right)}{\lambda\left(1-p_{1}^{i}\right)-a_{n} \theta / n}\right] \\
& =: H_{n}(\theta) .
\end{aligned}
$$

Similarly, for $\theta<0$, the above inequality should be reversed. According to (18) and $\log (1+x)=x-x^{2} / 2+o\left(x^{2}\right)$ as $x \longrightarrow 0$, one has

$$
\begin{aligned}
\log I_{n}(\theta) & =\frac{-\theta a_{n}}{n \lambda} \sum_{i=1}^{n} \frac{1}{1-p_{1}^{i}}-n \log \left(1-\frac{a_{n} \theta}{n \lambda}\right) \\
& =\frac{-\theta a_{n}}{\lambda}\left(1+b_{n}\right)+\frac{\theta a_{n}}{\lambda}+\frac{\theta^{2} a_{n}^{2}}{2 n \lambda^{2}}+o\left(\frac{a_{n}^{2}}{n}\right) \\
& =\frac{-\theta a_{n} b_{n}}{\lambda}+\frac{\theta^{2} a_{n}^{2}}{2 n \lambda^{2}}+o\left(\frac{a_{n}^{2}}{n}\right),
\end{aligned}
$$

where $b_{n}$ belongs to $\left[0,1 /\left(n\left(1-p_{1}^{2}\right)\right)\right]$. Hence,

$$
\lim _{n \longrightarrow . \infty} \frac{n}{a_{n}^{2}} \log I_{n}(\theta)=\frac{\theta^{2}}{2 \lambda^{2}},
$$

and, similarly,

$$
\lim _{n \rightarrow \infty} \frac{n}{a_{n}^{2}} \log H_{n}(\theta)=\frac{\theta^{2}}{2 \lambda^{2}} .
$$

Equation (39) is followed by (43), (48), (50), and (51). Consequently,

$$
\begin{aligned}
\Delta^{*}(x) & =\sup _{\theta \in R}\{\theta x-\Delta(\theta)\}=\sup _{\theta \in R}\left\{\theta x-\frac{\theta^{2}}{2 \lambda^{2}}\right\} \\
& =\frac{\lambda^{2} x^{2}}{2} .
\end{aligned}
$$

In addition, for any $\theta \in R, \Delta^{\prime}(\theta)=\theta / \lambda^{2}$; so the range of $\Delta^{\prime}(\theta)$ is $R$, which means $\mathscr{F}=R$; see Lemma 2.3.9 of [7].

Proof of Theorem 2. Note that the set of exposed points of $\Delta^{*}$ is $R$; Theorem 3 follows from Lemma 7 and the Gärtner-Ellis theorem.

\section{Appendix}

\section{The Gärtner-Ellis Theorem}

Consider a stochastic process $\left\{S_{n}\right\}_{n \geq 0}$, where $S_{n}$ possesses the law $\nu_{n}$ and logarithmic moment generating function $\Lambda_{n}(\theta):=$ $\log E\left(e^{\theta S_{n}}\right)$.
Assumption A. For each $\theta \in R$ and $0<b_{n} \longrightarrow \infty$, the logarithmic moment generating function, defined as the limit

$$
\Lambda(\theta)=\lim _{n \longrightarrow \infty} \frac{1}{b_{n}} \Lambda_{n}\left(b_{n} \theta\right)
$$

exists as an extended real number. Further, the origin belongs to the interior of $\{\theta: \Lambda(\theta)<\infty\}$.

Definition. Let $\Lambda^{*}$ be the Fenchel-Legendre transform of $\Lambda$. $y \in R$ is an exposed point of $\Lambda^{*}$ if for some $\theta \in R$ and all $x \neq y$ it is verified that $\theta y-\Lambda^{*}(y)>\theta x-\Lambda^{*}(x) . \theta$ in the previous equation is called an exposing hyperplane.

Let $\mathscr{E}$ be the set of exposed points of $\Lambda^{*}$ whose exposing hyperplane belongs to the interior of $\{\theta: \Lambda(\theta)<\infty\}$. The following lemma is the Gärtner-Ellis theorem in large deviation theory; see [7] page 44.

Lemma A.1. Let Assumption A holds.

(a) For any closed set $F$,

$$
\limsup _{n \rightarrow \infty} \frac{1}{b_{n}} \log \nu_{n}(F) \leq-\inf _{x \in F} \Lambda^{*}(x) .
$$

(b) For any open set $G$,

$$
\liminf _{n \longrightarrow \infty} \frac{1}{b_{n}} \log v_{n}(G) \geq-\inf _{x \in G \cap \mathscr{E}} \Lambda^{*}(x) .
$$

\section{Data Availability}

No data were used to support this study.

\section{Conflicts of Interest}

The authors declare that there is no conflict of interest regarding the publication of this paper.

\section{Acknowledgments}

This research was partially supported by National Natural Science Foundation of China (Grant No. 11601260).

\section{References}

[1] T. W. Epps, "Stock prices as branching processes," Communications in Statistics. Stochastic Models, vol. 12, no. 4, pp. 529-558, 1996.

[2] J. P. Dion and T. W. Epps, "Stock prices as branching processes in random environments: estimation," Communications in Statistics-Simulation and Computation, vol. 28, no. 4, pp. 957-975, 1999.

[3] Z. Gao and Y. Zhang, "Limit theorems for a supercritical Poisson random indexed branching process," Journal of Applied Probability, vol. 53, no. 1, pp. 307-314, 2016.

[4] Z. Gao and W. Wang, "Large deviations for a Poisson random indexed branching process," Statistics \& Probability Letters, vol. 105, pp. 143-148, 2015.

[5] K. V. Mitov and G. K. Mitov, "Subcritical randomly indexed branching processes," Pliska Studia Mathematica Bulgarica, vol. 20, pp. 155-168, 2011. 
[6] G. K. Mitov, K. V. Mitov, and N. M. Yanev, "Critical randomly indexed branching processes," Statistics \& Probability Letters, vol. 79, no. 13, pp. 1512-1521, 2009.

[7] A. Dembo and O. Zeitouni, Large Deviations Techniques and Applications, vol. 38, Springer, New York, NY, USA, 2nd edition, 1998.

[8] K. L. Chung, Markov Chains with Stationary Transition Probabilities, Springer, New York, NY, USA, 2nd edition, 1967.

[9] P. Hall and C. C. Heyde, Martingale Limit Theory and Its Application, Academic Press, New York, NY, USA, 1980.

[10] T. S. Ferguson, A Course in Large Sample Theory, Chapman \& Hall, London, UK, 1996. 


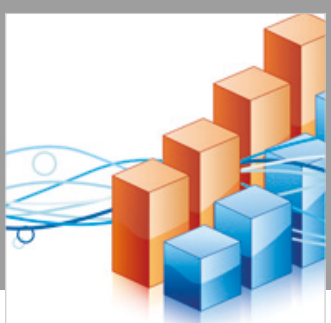

Advances in

Operations Research

\section{-n-m}
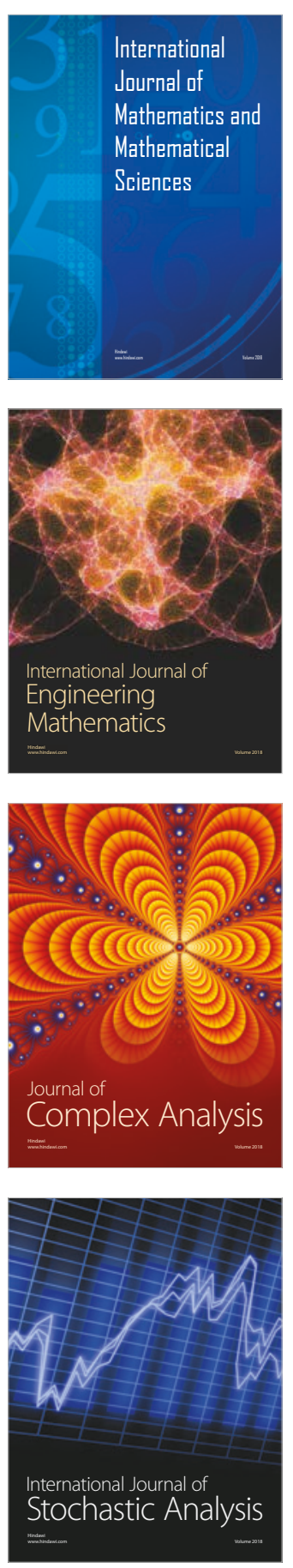
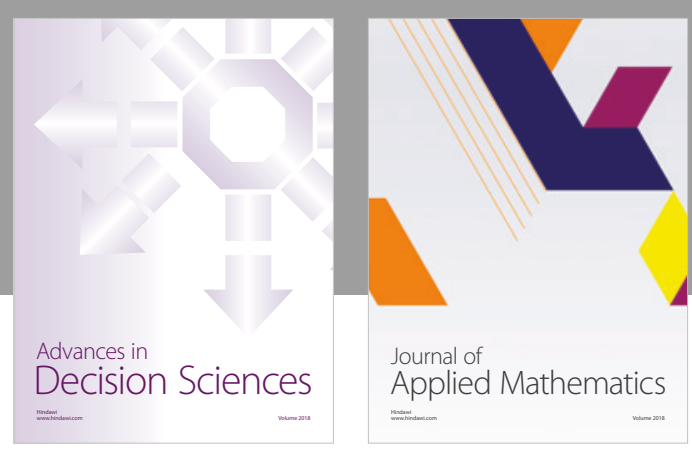

Journal of

Applied Mathematics
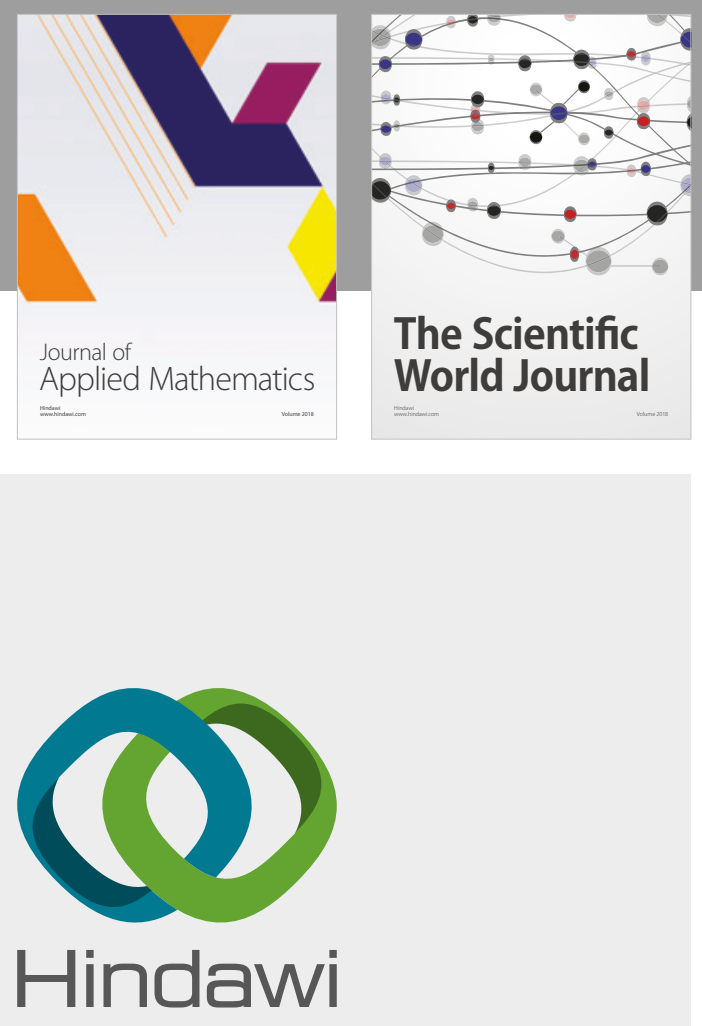

Submit your manuscripts at

www.hindawi.com

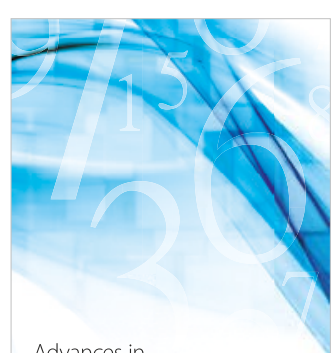

Advances in
Numerical Analysis
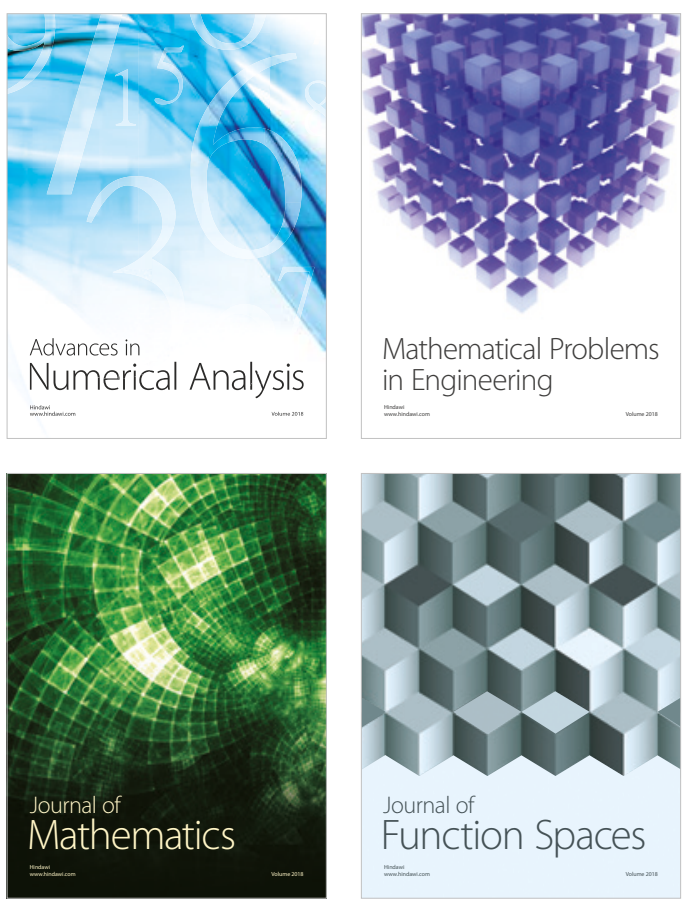

Mathematical Problems in Engineering

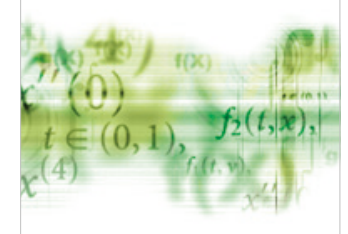

International Journal of

Differential Equations

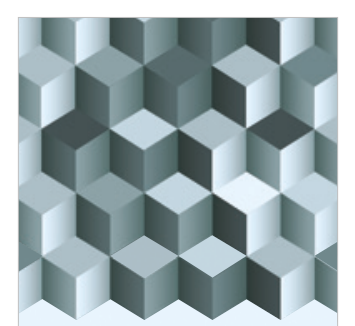

Journal of

Function Spaces

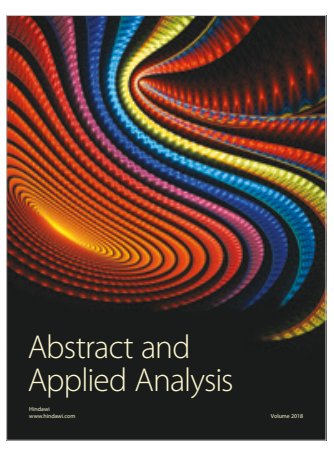

The Scientific

World Journal

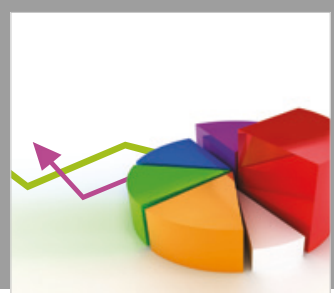

Journal of

Probability and Statistics
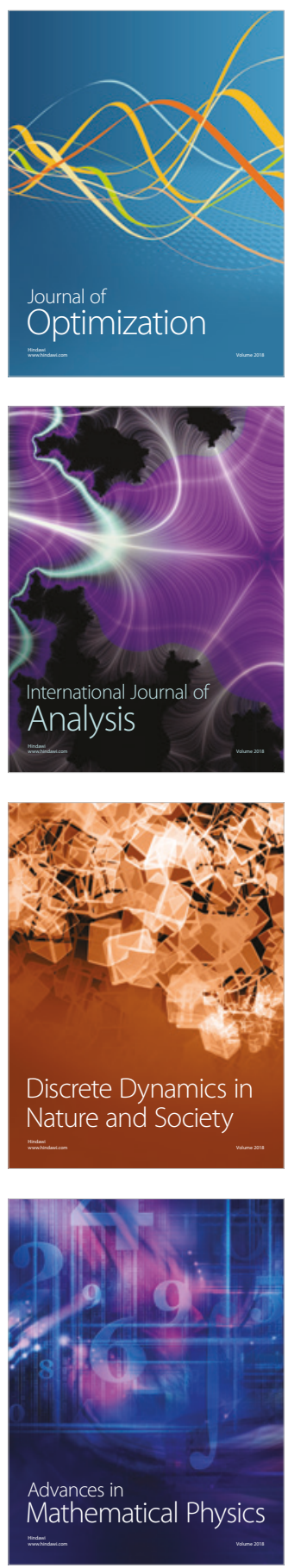